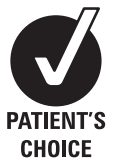

CHOICE

\title{
Social dysfunctioning after mild to moderate first- ever stroke at vocational age
}

\author{
M Hommel, ${ }^{1,2}$ S Trabucco-Miguel, ${ }^{1}$ S Joray, ${ }^{3}$ B Naegele, ${ }^{1,4}$ N Gonnet, ${ }^{2}$ A Jaillard ${ }^{1,4}$
}

${ }^{1}$ Stroke Unit, University Hospital-Joseph Fourier University, Grenoble, France; ${ }^{2}$ INSERM CIC 003, University Hospital Grenoble, Grenoble, France; ${ }^{3}$ Department of Neurology, University Hospital, Lausanne, Switzerland;

${ }^{4}$ Research Center, Grenoble Institute of Neuroscience Unit UMRS Inserm UJF, Grenoble, France

Correspondence to: Dr M Hommel, Stroke Unit, University Hospital, Grenoble, BP 217-38043 Grenoble Cedex 9, France;

marc.hommel@ujf-grenoble.fr

Received 14 July 2008 Revised 23 October 2008 Accepted 24 October 2008 Published Online First 14 November 2008

\begin{abstract}
Background: With improvements in stroke treatments, the number of patients with dramatic recovery is increasing. However, many of them are still complaining of difficulties in returning to work and every day activities. The aim was to assess work and social dysfunctioning in patients with minor to moderate stroke and explore its contributing factors.
\end{abstract}

Methods: Consecutive patients were prospectively included at a median 7 months after a first-ever stroke. Scores on the Work and Social Adjustment Scale (WSAS), a generic self-reported scale for assessing social functioning, were correlated with scores on the National Institutes of Health Stroke Scale (NIHSS), activities of daily living, Hospital Anxiety and Depression scale (HAD) and MMSE, lowa Scale of Personality Changes and return to work at 1 year.

Results: Among the 84 included patients (mean age 43.5 years), 57 (68\%; 95\% Cl 57 to $78 \%$ ) complained of significant perturbation of functioning attributed to stroke. WSAS was highly significantly related to modified Rankin scale, daily living activities, lowa Scale of Personality Changes and return to work at 1 year. Using ordinal logistic regression, the contributors to WSAS were initial neurological severity (NIHSS at admission), HAD and MMSE.

Conclusions: The study showed that up to $68 \%$ of our patients complained of significant work and social dysfunction due to stroke, despite a good clinical outcome. This self-estimation was correlated to external validation criteria, stressing the high burden of stroke from the patient's viewpoint. Moreover, when compared across diseases, social dysfunctioning after mild stroke was as important as in other major disabling diseases.

Major improvements in stroke prognosis have been achieved by stroke units implementation and increasing the routine use of thrombolysis. The number of survivors with dramatic recovery resulting in minor to moderate sequelae is therefore increasing. However, despite good motor and cognitive recovery, many of them are still reporting difficulties in social functioning (SF) leading to difficulties in returning to work $^{1-3}$ and to social and familial disturbances. ${ }^{45}$ Assessing these difficulties and identifying the determinants becomes a major issue to help the patients overcome their handicap.

The purpose of our study was to assess the frequency and the determinants of SF from the patient's viewpoint, several months after a mild to moderate stroke. At that time, patients had returned to their previous daily life, and the major part of functional recovery had occurred. Moreover, we focused on the subpopulation of patients in vocational age (range 18 to 65 years) who had apparently no severe remaining handicap. As the patient's opinion is becoming a valuable outcome, ${ }^{6}$ we assessed patient's self-estimation of how stroke had changed their SF. In parallel, we evaluated the patients' SF provided by the families, medical evaluation and return to work. To our knowledge, this is the first study focused on the patient's self-estimated SF after mild stroke.

Several scales allow SF to be assessed after stroke. The stroke impact scale ${ }^{7}$ measures SF but only as a part of the participation domain, which counts for one of the eight domains of this scale. Global scales are usually validated as a sum and not for using items separately. Moreover, strokespecific scales do not allow any comparisons across diseases. Conversely, the Work and Social Adjustment Scale (WSAS) ${ }^{8}$ is simple, quick, selfreported and focused on SF. The WSAS has been validated and has a good internal consistency and good sensitivity to the patient's condition modifications, such as improvement under therapy. ${ }^{89}$ Moreover, as a generic scale the WSAS allows comparisons across various disorders. It has been used in psychiatric disorders such as depression, ${ }^{8}$ obsessive compulsive disorders, ${ }^{8}$ phobia ${ }^{10}$ and bipolar disorders, ${ }^{11}$ after head trauma ${ }^{12}$ and in cardiac diseases.

\section{METHODS AND SUBJECTS Inclusion criteria}

This prospective cohort included patients admitted consecutively to the stroke unit with a first-ever stroke providing they met the following criteria: a recent 6 (SD 3)-month stroke demonstrated on imaging, and a vocational age from 16 years to 65 years. Patients were not eligible if they presented with transient ischaemic attack, National Institutes of Health Stroke Scale (NIHSS) $>4$ at study time, severe aphasia, alexia, non-compensated hemianopia, neglect, decreased visual acuity, illiteracy, psychiatric disorder, symptomatic prior stroke or silent stroke on imaging or other neurological disease. A score of $>23 / 30$ was required for the Mini Mental State Examination $(\mathrm{MMSE})^{13}$ to exclude patients with dementia or who were not able to sustain the whole set of tests. A modified Rankin scale ${ }^{14} \leqslant 2$ was chosen to exclude patients who had severe limitations of physical activities. The Medical Ethics Committee (CCPRB) of the institution approved the study. We obtained signed informed consent from all the subjects.

\section{Clinical evaluation}

At inclusion in the study, we evaluated handedness, ${ }^{15}$ depression using the Beck depression inventory ${ }^{16}$ 
(BDI) and hospital anxiety and depression scale (HAD), ${ }^{17}$ and performed the MMSE. Neurological deficit was assessed using the $\mathrm{NIHSS}^{18}$ at admission in the stroke unit in the acute period of the stroke and at the time of evaluation in the study. Handicap was scored using the modified Rankin scale. ${ }^{14}$ For assessing activities of daily living, we used the Self-maintaining and Instrumental Activities of Daily Living scale ${ }^{19}$ (SI-ADL). SI-ADL scale explores eight items rated from 0 to 1 : using the telephone, shopping, cooking, housework, laundry, transportation, medication and finances. SI-ADL was used as a total score, 8 representing normal activity.

Self-estimated work and social dysfunctioning was assessed with the WSAS, which explores five domains: ability to work, home management, social leisure, private leisure and ability to form and maintain close relationships with others. The WSAS is based on the subject's estimated capacities and resources and not on the subject's actual performance ("what I have done"). The consequences of an identified condition such as a stroke are assessed, since each question begins with "because of my condition ' $x$ ' stroke my activity ' $y$ ' is impaired". The five items of the WSAS were rated from 0 (no impairment) to 8 (very severely impaired), and the scores obtained for each item are summed in a total score. A score of 0 reflects normal functioning, and a score of 40 the worst score possible. We applied a similar categorisation of WSAS into three categories as those reported in other diseases. ${ }^{8}$ A score $\geqslant 20$ suggested moderately severe to severe impairment, from 10 to 19 significant dysfunctioning, and less than 10 a subclinical dysfunctioning.

We also measured SF with a complementary approach, provided by the families, medical evaluation and professional status.

1. To obtain an assessment of the behavioural and social dysfunctioning, spouses or a family member filled the lowa Scale of Personality Changes (ISPC), which estimates affective, behavioural and social disturbances that may occur after a brain lesion, and assesses the extent of changes from premorbid levels. ${ }^{20}$ Twenty-nine items explored behaviour, behavioural control, goal-directed behaviour, decision-making, emotional expression, interpersonal relations and insight, on a scale from 1 to 7 . A rating of 1 reflected excellent, and a rating of 7 reflected severe disability. The difference between prestroke and current ratings provided a measure of the extent of the changes. The two assessments were made when assessing the WSAS, and we used the mean of the changes within the 29 items as a measure of personality changes.

2. For medical evaluation, experienced stroke neurologists in charge of the patients rated globally SF into three categories unaware of the WSAS rating of the patients: no social dysfunctioning, mild to moderate social dysfunctioning, severe social dysfunctioning.

3. We collected information on profession and working status before stroke, at inclusion and 1 year after stroke. Moreover, the occurrence of conjugal life problems since stroke was recorded as present or absent.

4. Neuropsychologists collected patient's WSAS ratings and assessed HAD and MMSE.

\section{Magnetic resonance imaging}

All patients underwent MRI including T2, FLAIR and DWI within the first week following stroke to confirm the diagnosis.

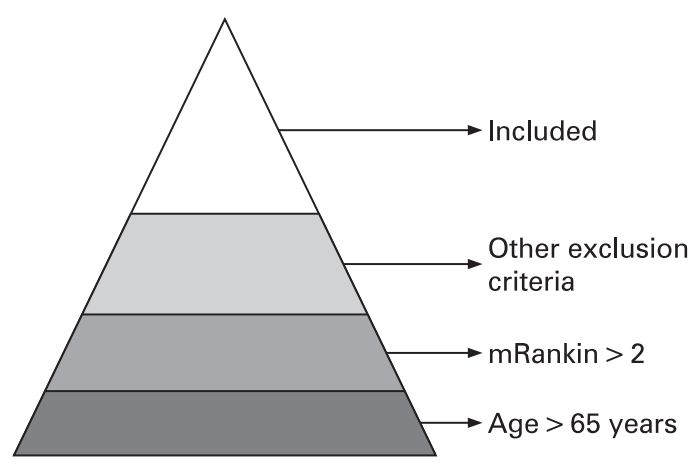

Figure 1 Selection of the patients. mRankin, modified Rankin.

\section{Data and statistical analysis}

For univariate comparisons, we used ANOVA, Kruskal-Wallis test, Fisher exact, $\chi^{2}$ test and Cuzick test for trends when appropriate. Internal validity of WSAS was assessed using the Cronbach alpha and principal-components analysis. We analysed statistical associations between WSAS and NIHSS, modified Rankin scale, SI-ADL, ISPC, global clinical assessment by stroke neurologists or return to work at 1 year.

We used ordinal regression modelling to approach which domains were expressed by the WSAS. Variables associated with WSAS at univariate analysis were removed stepwise with a level set at $p>0.2$. The parallel regression assumption of the ordinal logistic model was assessed with the Brant test. ${ }^{21}$ Confidence intervals were computed using bootstrapping ${ }^{22}$ with 2050 replications. The level of significance was chosen at 0.05 . Statistical analyses were performed using STATA 9.2 (STATA Corp, College Station, Texas).

Table 1 Demographic and clinical characteristics of the patients

\begin{tabular}{lc}
\hline Demographics & Total (N = 84) \\
\hline Mean age (SD) & $43.5(12.2)$ \\
Males, N (\%) & $43(51.2)$ \\
Females, N (\%) & $41(48.8)$ \\
Education primary and secondary, N (\%) & $43(51.2)$ \\
Education university level, N (\%) & $41(48.8)$ \\
Clinical findings & \\
Handedness (Edinburgh score) >90\% & $77(92.7)$ \\
Beck Depression Inventory mean (SD) & $11.2(9.5)$ \\
HAD mean (SD) & $14.5(7.1)$ \\
Mini-Mental State Examination median (25\% to 75\% IO) & $29(27$ to 30$)$ \\
NIHSS mean (SD)—on admission & $5.8(4.3)$ \\
NIHSS mean (SD)-at study time & $0.9(1.2)$ \\
Modified Rankin, median (25th to 75th IQ)—at study time & $2(1$ to 2$)$ \\
Delay S-E months, mean (SD; range) & $7.2(3.9 ; 3$ to 10$)$ \\
Taking antidepressant drug, N (\%) & $35(42 \%)$ \\
Description of stroke & \\
Infarction, N (\%) & $79(0.94)$ \\
Haematoma, N (\%) & $5(0.06)$ \\
Right-hemispheric infarct, N (\%) & $36(42.8)$ \\
Left-hemispheric infarct & $30(35.7)$ \\
Bilateral hemispherical infarcts & $7(8.3)$ \\
Brainstem, cerebellum & $11(13.1)$ \\
\hline
\end{tabular}

Delay S-E, Delay from stroke to evaluation; HAD, Hospital Anxiety and Depression scale; IQ, interquartile; NIHSS at study time, National Institutes of Health Stroke Scale at inclusion in the study, mean 7.2 months after stroke; NIHSS on admission, National Institutes of Health Stroke Scale at admission with acute stroke in the stroke unit. 
Table 2 Modified Rankin at inclusion versus Work and Social Adjustment Scale (WSAS)

\begin{tabular}{lcccl}
\hline $\begin{array}{l}\text { Modified Rankin } \\
\text { at inclusion }\end{array}$ & WSAS $<\mathbf{1 0}$ & $\mathbf{1 0} \leqslant$ WSAS $<\mathbf{1 9}$ & WSAS $\geqslant \mathbf{2 0}$ & Total N (\%) \\
\hline 0 N (\%) & 8 & 2 & 2 & $12(14.3)$ \\
1 N (\%) & 12 & 6 & 6 & $24(28.6)$ \\
2 N (\%) & 7 & 16 & 25 & $48(57.1)$ \\
Total N (\%) & $27(32.1)$ & $24(28.6)$ & $33(39.3)$ & 84 \\
\hline
\end{tabular}

\section{RESULTS}

We included 84 patients of the 410 (20\%; 95\% CI 17\% to $25 \%$ ) patients with stroke admitted to the stroke unit during that period of 15 months (fig 1). The demographic and clinical characteristics are listed in table 1.

Fifty-seven patients (68\%; $95 \%$ CI $57 \%$ to $78 \%$ ) complained of at least significant dysfunctioning in SF attributed to stroke based on WSAS. The distribution of our population was almost equivalent within the three categories of the WSAS (table 2).

The Cronbach alpha of the WSAS was 0.87 . The first factor of principal-component analysis explained $55 \%$ of the variance with a group of four close items (ability to work, home management, social leisure, private leisure). The second factor explaining $23 \%$ of the variance was almost exclusively represented by the ability to form and maintain close relationships with others.

Self-estimated dysfunctioning was not significantly related to NIHSS at the time of WSAS examination (Fisher exact; $p=0.65)$, but presented a trend with the NIHSS at admission to the stroke unit (Kruskal-Wallis test; $p=0.09$ ). The modified Rankin scale at examination (Fisher exact; $p=0.001$; table 2) and SI-ADL (Kruskal-Wallis test; $p=0.0001$; fig 2) were significantly related to WSAS. The global assessment done by the stroke neurologists was significantly related to WSAS (Fisher exact; $\mathrm{p}=0.007$; table 3 ).

The relation between WSAS rating and working status at 1 year was highly significant (Fisher exact $p<0.000$; table 4).

The mean differences in ISPC were significantly related to WSAS (ANOVA $F=4.23 ; p=0.02$; fig 3 ). Conjugal difficulties were associated with medical evaluation (Pearson $\chi^{2}=22.4$; $\mathrm{p}<0.001$ ) and ISPC (ANOVA $\mathrm{F}=3.45 ; \mathrm{p}=0.04$ ) but not with the WSAS (Fisher exact; $p=0.22$ ).

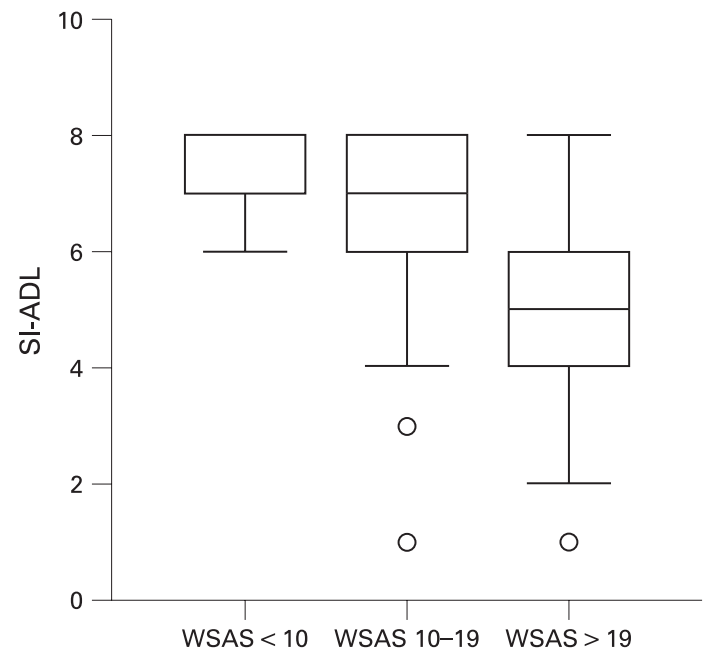

Figure 2 Self-maintaining and Instrumental Activities of Daily Living Scale (SI-ADL) according to Work and Social Adjustment Scale (WSAS) categories.
When tested in univariate analysis, the WSAS was not significantly related to handedness (Pearson $\chi^{2} ; p=0.97$ ), hemispheric lateralisation of stroke (Fisher exact; $p=0.66$ ), age (ANOVA $\mathrm{F}=1.00 ; \mathrm{p}=0.37)$, gender (Pearson $\chi^{2} ; \mathrm{p}=0.23$ ) or level of education (Pearson $\chi^{2} ; p=0.06$ ). The WSAS was significantly associated with MMSE (Cuzick non-parametric test for trend; $p=0.001$ ), BDI (Kruskal-Wallis test; $p=0.001$ ) and HAD (ANOVA; $F=16.01, p<0.0000$ ). Items of the BDI allowed us to test the relations between frequent clinical complaints and SF. Fatigue (Fisher exact; $p=0.046$ ), loss of concentration (Fisher exact; $\mathrm{p}<0.000$ ) and irritability (Fisher exact; $p=0.01$ ) were significantly related to WSAS

Using ordinal logistic regression, the Brant test confirmed that parallel regression assumption was respected $\left(\chi^{2}=0.57\right.$; $\mathrm{p}>0.90)$. Three variables were selected in the model, NIHSS at admission, MMSE at examination and HAD (table 5).

\section{DISCUSSION}

The included patients represented those with lower age and lower residual disability, as shown on fig 1 . The surfaces of each polyhedron constituting the pyramid of the selection of the patients (fig 1) are proportional to the percentages of the subpopulations of acute stroke admitted to the stroke unit. In our population, the mean WSAS was 16.1 (SD 10.0; median 16.5), corresponding to significant social dysfunctioning. Among the 84 patients, SF evaluated by the WSAS was moderately impaired in $28.6 \%$ and severely impaired in $39.3 \%$.

Moreover, as a generic scale, WSAS allows comparisons across diseases. When comparing WSAS scores in our patients with stroke (mean 16.1, SD $=10.0$, median $=16.5$ ) with reported WSAS scores in unipolar depression before treatment (mean $17.8)^{8}$ and in bipolar disorders in remission (median 14), ${ }^{10}$ the WSAS scores were of the same order of magnitude. Considering that unipolar depression has been ranked within the top 10 of

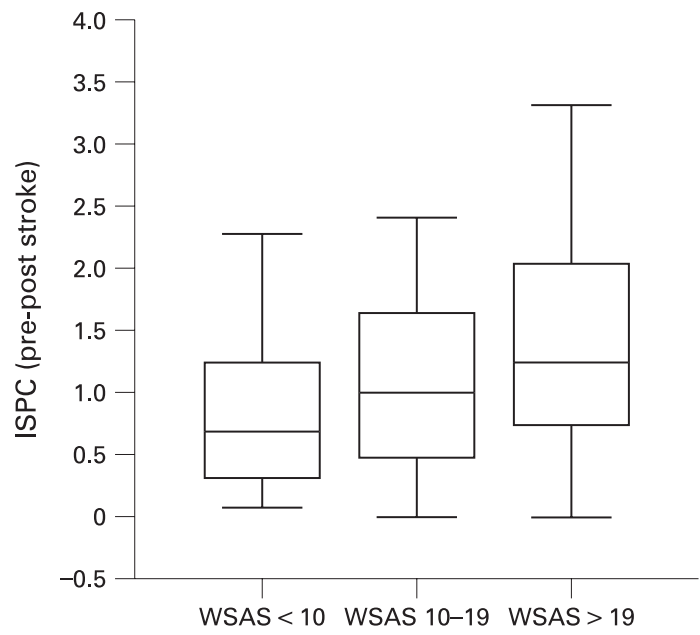

Figure 3 lowa Scale of Personality Changes (ISPC) according to Work and Social Adjustment Scale (WSAS) categories. 
Table 3 Neurologist's rating versus patient's Work and Social Adjustment Scale (WSAS)

\begin{tabular}{lllll}
\hline $\begin{array}{l}\text { Neurologist's assessment } \\
\text { of work and social dysfunctioning }\end{array}$ & WSAS $<\mathbf{1 0}$ & $\mathbf{1 0} \leqslant$ WSAS $<\mathbf{1 9}$ & WSAS $\geqslant \mathbf{2 0}$ & Total N (\%) \\
\hline 1 no (N\%) & 15 & 12 & 7 & $34(41.5)$ \\
2 significant mild (N\%) & 8 & 6 & 7 & $21(25.6)$ \\
3 moderate to severe (N\%) & 4 & 5 & 18 & $27(32.9)$ \\
Total (N\%) & $27(32.9)$ & $23(28.0)$ & $32(39.0)$ & 82 \\
\hline
\end{tabular}

Table 4 Return to work 1 year after stroke

\begin{tabular}{lllcc}
\hline Profession & WSAS $<\mathbf{1 0}$ & $\mathbf{1 0} \leqslant$ WSAS $<\mathbf{1 9}$ & WSAS $\geqslant \mathbf{2 0}$ & Total N (\%) \\
\hline Without profession before stroke & 2 & 4 & 2 & $8(10.5)$ \\
No return to work & 3 & 6 & 22 & $31(40.8)$ \\
Part time work & 4 & 3 & 1 & $8(10.5)$ \\
Other than before, full-time work & 2 & 1 & 0 & $3(4.0)$ \\
Same as before, full-time & 11 & 10 & 5 & $26(34.2)$ \\
Total (N\%) & $22(28.9)$ & $24(31.6)$ & $30(39.5)$ & 76 \\
\hline
\end{tabular}

WSAS, Work and Social Adjustment Scale.

disability-adjusted life years (DALYs) in the world, in terms of disease-related handicap ${ }^{23}$ these findings highlight the burden of stroke, even with a good clinical outcome.

Scales commonly used in stroke such as the NIHSS ${ }^{18}$ measure neurological deficit, or basic daily activities with the Barthel index. ${ }^{24}$ The modified Rankin scale ${ }^{14}$ provides a crude rating considered as a quick scoring of handicap. They are widely used, either alone $e^{25}$ or in combination ${ }^{26}$ in clinical trials ${ }^{27}$ and in routine practice, but they have obvious limitations in higherorder and social functioning. Focused on house and social activities, the Frenchay activity index ${ }^{28}$ and the Self-maintaining and Instrumental Activities of Daily Living ${ }^{19}$ (SI-ADL) measure what the patient is doing. The extensive and validated quality of life (QOL) questionnaire SF-36 explores many domains such as physical functioning, physical role, bodily pain, general health, vitality, SF, emotional role and mental health. ${ }^{29}$ However, generic tools assessing quality of life are timeconsuming, and questionnaires not focused on the specific problems reported by the patients do not arouse enough motivation to be filled in diligently, and so tools designed specifically for stroke have been created..$^{30-32}$ The SS-QOL, ${ }^{33}$ for example, showed a higher sensitivity to meaningful changes ${ }^{34}$ than the generic SF-36. ${ }^{29}$ However, when testing the clinical meaning of the stroke adapted Sickness Impact Profile (SASIP30) ${ }^{35}$ and the generic SIP, they primarily reflect aspects of physical functioning, the psychosocial dimension of both versions remaining largely unexplained. ${ }^{36}$ EuroQol is simple and measures aspects of quality of life; however, social and work functioning are approached only in the "usual activities" item. $^{37}$ Furthermore, these scales and indexes are rated by doctors or nurses or are questionnaires based on the evaluation of the patient's capabilities, "what he is able to do" or "what he is doing," and do not account for the patient's viewpoint. Conversely, the WSAS assessed the patient's self-estimation of how stroke had changed their SF.

The rate of SF dysfunctioning may be considered as surprisingly high, relative to the clinical findings (table 1). First, our patients were young (43.5 years). Although age was associated with a better outcome, a recent study has reported that age was inversely associated with mental and physical health at 1 year poststroke, which was attributed to higher expectations of health and recovery in young patients. ${ }^{38}$ Actually, our patients had globally mild to moderate stroke, which is accounted for by the low NIHSS (mean 5.8) at admission with acute stroke, and a good clinical outcome, accounted for by the mean NIHSS of 0.9 and modified Rankin scale of 1.4 on the day of the WSAS assessment. Second, the low depression score (mean BDI within the minimal range and mean $\mathrm{HAD}$ score was at the edge of depression) should have led to a better self-estimated SF. However, $42 \%$ were taking antidepressant drugs prescribed for depression symptoms. Third, the high MMSE at 7.1 months (median 29) did not indicate a significant cognitive impairment or dementia that might account for social dysfunctioning. However, the $25 \%$ of the patients who had a MMSE <27 may have cognitive difficulties, in either episodic memory (free recall of the three words) or working memory (maintenance and calculation during the five consecutive subtractions). Therefore, although our population represents the subset of mild handicapped patients with stroke under 65 years old, the high rate of social dysfunction highlights the fact that SF was perceived to be problematic by these patients with stroke.

However, it could be argued that patients may have overestimated their social dysfunctioning. When testing selfestimated social dysfunctioning with other scales, the association between WSAS and modified Rankin scale was highly significant, despite 16 (19\%; 95\% CI 11.1 to 29.4\%) patients with an excellent score (modified Rankin scale of 0 to 1 ) complaining of significant social dysfunctioning. The strong association between the self-assessed severity in WSAS and SI$\mathrm{ADL}$ representing the activities done by the patient in daily living functioning was clearly illustrated in fig 2, highlighting the fact that the patient's self-assessment reflects everyday activities. A similar correlation with ISPC (fig 3) confirms that self-estimation was in agreement with the familys' ratings of the patient's behaviour. There was also a strong association between WSAS and the stroke neurologist's ratings, thus confirming the fact that the medical evaluation and the

Table 5 Ordinal logistic model of the three variables predicting the Work and Social Adjustment Scale

\begin{tabular}{lllll}
\hline & Odds ratio & Bootstrap SE & $\mathbf{p}>|\mathbf{z}|$ & $\mathbf{9 5 \%} \mathbf{C l}$ \\
\hline NIHSS Day 0 & 1.13 & 0.07 & 0.046 & 1.002 to 1.276 \\
MMSE & 0.60 & 0.10 & 0.002 & 0.440 to 0.826 \\
HAD & 1.26 & 0.07 & 0.000 & 1.134 to 1.497
\end{tabular}

HAD, Hospital Anxiety and Depression scale; MMSE, Mini-Mental State Examination; NIHSS Day 0, National Institutes of Health Stroke Scale at admission. 
patient's self-estimation are closely linked. The highly significant link between working status and social dysfunctioning was confirmed when assessing patients returning to work 1 year after stroke. Moreover, a WSAS $\geqslant 20$ at 7 months was predictive of a major risk of not returning at work, whatever its modality (risk ratio $=3.49$; 95\% CI 1.90 to 6.41 ). Therefore, these multiple external evaluations allowed us to validate the patient's self-estimation and to consider the WSAS as a reliable measure of the patient's SF.

SF has been recently evaluated using quality-of-life scales (SF36). Ethnicity, ischaemic heart disease and cognitive impairment were independent factors of mental health, a subscore including SF. ${ }^{38}$ Other long-term outcome studies have reported age, depression, cognitive impairment, disability, aphasia and poor social network to be associated with a poor quality of life. When exploring the factors contributing to WSAS using ordinal logistic regression, the NIHSS at admission, HAD and MMSE explained the WSAS. While bivariate statistics showed only a trend toward significance for the NIHSS, multivariate modelling kept the NIHSS at admission in the model after adjustment for depression and anxiety, because severe strokes predicted mostly poor SF. HAD included the psychological components depression and anxiety, which are the most robust predictors of inadequate SF and have been reported as predictive factors of recovery in many studies. ${ }^{39}$ Mood can be viewed as the affective or emotional SF corresponding to the second factor of the principal-component analysis of the WSAS. MMSE, which summarised cognitive functions, was the third contributor of SF. The role of cognitive impairment in recovery and quality of life has been well documented. Our findings are consistent with other studies, although our population was younger and less impaired regarding physical and cognitive aspects. The fatigue, loss of concentration and irritability items were also associated with SF, supporting the idea that cognition and mood are leading factors of SF.

The assessment of SF was performed a median of 7 months after stroke, which corresponds to a short delay in comparison with other studies. However, at that time, most of the recovery has been achieved. Furthermore, we wished to avoid interfering events and focused the evaluation of SF when patients' selfestimation could be reliably related to their stroke. However, information on possible evolution of the WSAS scores with time should be documented in further studies.

This study showed that self-assessed work and social dysfunctioning provides valuable information on SF after stroke. Social dysfunctioning seemed to be frequent months after stroke, stressing the burden for mild to moderate stroke in young and non-demented patients. Finally, initial neurological severity, mood and cognitive functions appeared to determine self-estimation of social dysfunctioning.

Acknowledgements: This study was supported by a grant of the DRRC of the University Hospital of Grenoble VASCO 2004 (academic grant).

Permission was obtained from Dr Marks to use WSAS.

Competing interests: None.

Ethics approval: Ethics approval was provided by Comité de Protection des Personnes se prêtant à la Recherche Biomédicale.

\section{REFERENCES}

1. Treger I, Shames J, Giaquinto S, et al. Return to work in stroke patients. Disabil Rehabil 2007;29:1397-403.

2. Alaszewski A, Alaszewski $\mathrm{H}$, Potter J, et al. Working after a stroke: survivors' experiences and perceptions of barriers to and facilitators of the return to paid employment. Disabil Rehabil 2007;29:1858-69.
3. Varona JF, Bermejo F, Guerra JM, et al. Long-term prognosis of ischemic stroke in young adults. Study of 272 cases. J Neurol 2004;251:1507-14.

4. Giaquinto S, Buzzelli S, Di Francesco L, et al. Evaluation of sexual changes after stroke. J Clin Psychiatry 2003;64:302-7.

5. Carod-Artal J, Egido J, González J, et al. Quality of life among stroke survivors evaluated 1 year after stroke: experience of a stroke unit. Stroke 2000;31:29953000.

6. Pound P, Tilling K, Rudd AG, et al. Does patient satisfaction reflect differences in care received after stroke? Stroke 1999;30:49-55.

7. Duncan PW, Wallace D, Lai SM, et al. The stroke impact scale version 2.0. Evaluation of reliability, validity, and sensitivity to change. Stroke 1999;30:2131-40.

8. Mundt JC, Marks IM, Shear MK, et al. The Work and Social Adjustment Scale: a simple measure of impairment in functioning. Br J Psychiatry 2002;180:461-4.

9. McLaughlin TJ, Aupont O, Bambauer KZ, et al. Improving psychologic adjustment to chronic illness in cardiac patients. The role of depression and anxiety. J Gen Intern Med 2005;20:1084-90.

10. Mataix-Cols D, Cowley AJ, Hankins M, et al. Reliability and validity of the work and social adjustment scale in phobic disorders. Compr Psychiatry 2005:46:223-8.

11. Fagiolini A, Kupfer DJ, Masalehdan A, et al. Functional impairment in the remission phase of bipolar disorder. Bipolar Disord 2005;7:281-5.

12. Edna TH, Cappelen J. Return to work and social adjustment after traumatic head injury. Acta Neurochir (Wien) 1987;85:40-3.

13. Folstein M, Folstein S, McHugh P. Mini-Mental State: A practical method for grading the cognitive state of patients for the clinician. J Psychiatr Res 1975;12:189-98.

14. van Swieten JC, Koudstaal PJ, Visser MC, et al. Interobserver agreement for the assessment of handicap in stroke patients. Stroke 1988;19:604-7.

15. Oldfield RC. The assessment and analysis of handedness: the Edinburgh inventory Neuropsychologia 1971;9:97-113

16. Aben I, Verhey F, Lousberg R, et al. Validity of the beck depression inventory, hospital anxiety and depression scale, SCL-90, and hamilton depression rating scale as screening instruments for depression in stroke patients. Psychosomatics 2002:43:386-93.

17. Zigmond AS, Snaith RP. The hospital anxiety and depression scale. Acta Psychiatr Scand 1983:67:361-70.

18. Brott T, Adams HP Jr, Olinger CP, et al. Measurements of acute cerebral infarction: a clinical examination scale. Stroke 1989;20:864-70.

19. Lawton MP, Brody EM. Assessment of older people: self-maintaining and instrumental activities of daily living. Gerontologist 1969;9:179-86.

20. Barrash J, Anderson S, Jones R. The lowa rating scales of personality change: reliability and validity. J Int Neuropsychol Soc 1997;3:27-8.

21. Brant R. Assessing proportionality in the proportional odds model for ordinal logistic regression. Biometrics 1990;46:1171-8.

22. Poi B. From the help desk: some bootstrapping techniques. Stata J 2004;4:312-28.

23. Mathers CD, Loncar D. Projections of global mortality and burden of disease from 2002 to 2030. PLoS Med 2006;3:2011-30.

24. Mahoney FI, Barthel DW. Functional evaluation: the Barthel Index. Md State Med J 1965;14:61-5.

25. The Multicenter Acute Stroke Trial-Europe Study Group. Thrombolytic therapy with streptokinase in acute ischemic stroke. N Engl J Med 1996;335:14550.

26. The National Institute of Neurological Disorders and Stroke rt-PA Stroke Study Group. Tissue plasminogen activator for acute ischemic stroke. N Engl J Med 1995;333:1581-7.

27. Ali M, Bath PM, Curram J, et al. The Virtual International Stroke Trials Archive. Stroke 2007;38:1905-10.

28. Wade DT, Legh-Smith J, Langton Hewer R. Social activities after stroke: measurement and natural history using the Frenchay Activities Index. Int Rehabil Med 1985; 7:176-81.

29. Ware JE, Sherbourne CD. The MOS 36-item short form health survey (SF-36) 1: conceptual framework and item selection. Med Care 1992;30:472-83.

30. Buck D, Jacoby A, Massey A, et al. Development and validation of NEWSOOL, the Newcastle Stroke-Specific Quality of Life Measure. Cerebrovasc Dis 2004;17:14352.

31. Bohannon RW, Maljanian R, Lee N, et al. Measurement properties of the short form (SF)-12 applied to patients with stroke. Int J Rehabil Res 2004;27:151-4.

32. Bohannon RW, Maljanian R, Landes M. Test-retest reliability of short form (SF)-12 component scores of patients with stroke. Int J Rehabil Res 2004;27:149-50.

33. Williams LS, Weinberger M, Harris LE, et al. Development of a stroke-specific quality of life scale. Stroke 1999;30:1362-9.

34. Williams LS, Weinberger M, Harris LE, et al. Measuring quality of life in a way that is meaningful to stroke patients. Neurology 1999;53:1839-43.

35. van Straten A, de Haan RJ, Limburg M, et al. A stroke-adapted 30-item version of the Sickness Impact Profile to assess quality of life (SA-SIP30). Stroke 1997;28:2155-61.

36. van Straten A, de Haan RJ, Limburg M, et al. Clinical meaning of the StrokeAdapted Sickness Impact Profile-30 and the Sickness Impact Profile-136. Stroke 2000;31:2610-15.

37. Dorman $\mathbf{P J}$, Waddell F, Slattery J, et al. Is the EuroOol a valid measure of healthrelated quality of life after stroke? Stroke 1997;28:1876-82.

38. Patel MD, McKevitt C, Lawrence $\mathrm{E}$, et al. Clinical determinants of long-term quality of life after stroke. Age Ageing 2007;36:316-22.

39. Townend BS, Whyte S, Desborough T, et al. Longitudinal prevalence and determinants of early mood disorder post-stroke. J Clin Neurosci 2007:14:429-34. 